\title{
INTERALLELIC RECOMBINATION AT THE his-I LOCUS IN NEUROSPORA CRASSA AND ITS GENETIC CONTROL*
}

\author{
ADRIENNE P. JESSOP \\ Blod Bonk, Bispegjerg Hospital, Copenhogen, NV, Denmark (temporary) \\ and \\ D. G. CATCHESIDE \\ Department of Genetics, John Curtin School of Medical Research, \\ Australian National University, Conberra, A.C.T., Austrolia
}

\section{INTRODUCTION}

Received I 7.xi.64

THE histidine-I gene of Neurospora crassa is situated in the right arm of chromosome $\mathrm{V}$ between amination and inositol (Strickland, Perkins and Veatch, 1959). It apparently controls the primary structure of the enzyme imidazole glycerol phosphate dehydrase, which catalyses the conversion of imidazole glycerol phosphate to imidazole acetol phosphate in the biosynthesis of histidine (Ames, I 960 ).

A large number of his-I mutants are known and some of these exhibit complementation. Initially the complementation matrix was thought to indicate a two-dimensional complementation map (Catcheside, I960), but this is now known to be an error (see pp. I7-19). Nevertheless, this was the reason for attempting to map the fine structure of his- I. Such mapping depends primarily upon the frequency of prototrophs found amongst ascospores produced by repulsion phase crosses between pairs of auxotrophs. It assumes that the frequency of prototrophs formed by such a cross is a measure of the physical distance between the actual sites at which the two auxotrophs differ from one another and so from the wild type gene. In consequence, it is expected that a series of allelic auxotrophs, all with point differences, would show prototroph frequencies that conformed to an additivity rule, namely that the frequency shown by a cross between any two auxotrophs would be the sum or the difference of the frequencies shown by crosses of these two auxotrophs with a third auxotroph. If this rule held and the frequencies were measured sufficiently accurately, the sites of mutation should be capable of arrangement in an unambiguous linear order.

However, two features of the his- $I$ data on prototroph frequencies led to difficulties. Firstly, many of the frequencies were very low and, secondly, as was later found, crosses involving different stocks of the same auxotrophs commonly gave widely different values. With further study, it became clear that the different values were mainly due to the

* The work was done in the Department of Microbiology, University of Birmingham. 
effects of genetic differences, principally at a locus designated rec, for recombination. Crosses, it was found, could be divided phenotypically into those with high and those with low frequencies of prototrophs, the factor of difference being about ten. Genotypically, the observations are explicable by assuming two allelic genes, $\mathrm{rec}$ and $\mathrm{rec}^{+}$, such that only crosses in which both parents were rec gave high frequencies of prototrophs, while crosses in which one or both of the parents were $\mathrm{rec}^{+}$gave low frequencies. In brief, the gene for low frequency is dominant to that for high frequency. Most of the stocks used may, with some confidence, be classified as rec or rec ${ }^{+}$.

Starting with a set of his- I mutants, all derived from one wild-type strain, crosses between them could be made only after outcrossing to introduce the other mating type, while further outcrosses were necessary to introduce the flanking markers. In these various manœuvres, the source and spread of so cryptic a genetic difference as the one described above will be hard to trace completely.

From the beginning of the work, reliance for mapping was not solely dependent upon prototroph frequency, but also on the distribution of the combinations of flanking markers, proximal and distal, amongst the prototrophs produced by crosses. If in a cross of the general type $A m^{1} B \times a m^{2} b$, where $m^{1}$ and $m^{2}$ are alleles whose recombination is being studied, while $A$ and $a$ and $B$ and $b$ are, respectively, the proximal and distal flanking markers, selection is made for $\mathrm{m}^{+}$ prototrophs, the flanking markers occur in four combinations. These are the two parental combinations $A B$ and $a b$ and the two recombinants $A b$ and $a B$. In Neurospora all four classes occur with substantial frequencies, where recombination of alleles is studied. A vast excess of one recombinant class, so characteristic of the distribution of flanking markers when prototrophic recombinants of two closely linked non-allelic genes are selected and indicative of the order of the gene differences, is not encountered. Nevertheless, the data do show some regularities. The parental classes are often unequal in size. The recombinant classes are often unequal in size. The directions of inequality of the parental and recombinant classes are correlated. The inequalities are usually not very great. It has been customary to assume that the high frequencies of the parental and the second recombinant classes are due to the conjoint occurrence of two or three crossovers showing a high incidence of negative interference. This is no more than a description of what the observations require in order to fit them to the chiasmatype theory of genetic recombination. We are not concerned here so much with the theory of recombination, as with what information about order of allelic differences may bc extracted from the data.

It is simplest to understand the criteria, if it is assumed that the recombination does occur by a process analogous to crossing-over (fig. I). Three relationships may be considered.

I. If the recombinant classes are compared, the order will be 
$A m^{1} m^{2} B$ if $a B$ is in the majority and will be $A m^{2} m^{1} B$ if $A b$ is in the majority.

2. If the proximal flanking alleles only are considered, the order will be $A m^{1} m^{2}$ if $a$ is in the majority and will be $A m^{2} m^{1}$ if $A$ is in the majority. In other words, the more frequent proximal flanking allele identifies the more distal of the $m$ allele differences; they entered the cross together.

3. If the distal flanking alleles only are considered, the order will be $m^{1} m^{2} B$ if $B$ is in the majority and will be $m^{2} m^{1} B$ if $b$ is in the majority. That is, the more frequent distal flanking allele identifies the more proximal of the $m$ allele differences; they entered the cross together.

The first criterion uses only the recombinant classes, whereas the second and third criteria also use the parental classes. Hence, although the second and third criteria are not wholly independent of the first, they do have the merit of using all of the progeny scored.
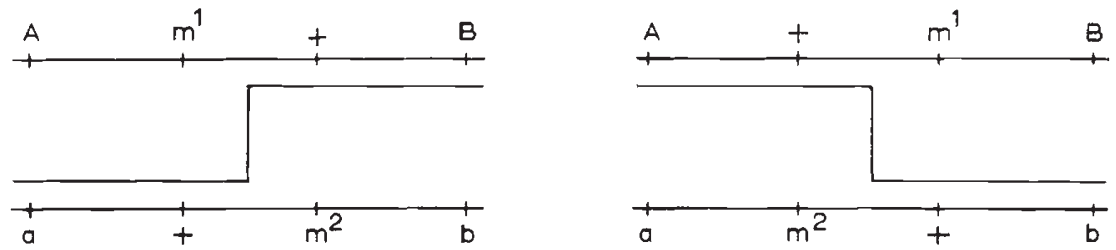

FIG. 1.-Diagrams to show the derivation of the criteria of order based upon the distribution of flanking genetic markers $(A$ and $B$ ) amongst prototrophs formed by allelic auxotrophs $\left(m^{1}\right.$ and $\left.m^{2}\right)$.

If any, or all, of these criteria lead to the derivation of a consistent order of a series of $m$ alleles, this order may be regarded as acceptable and meaningful. Consistency may be expected to hold between the three criteria applied to one pair of alleles and between a series of pairs of a set of aHeles considered with respect to one criterion. Inconsistency can be considered significant only if the order inferred is based upon a significant difference in frequency between the classes compared. The his-I data, as will be seen, mostly show rather small frequency differences, which are insignificant and therefore ambiguous.

It is convenient to distinguish between the two parental classes ( $P_{1}$ and $P_{2}$ ) and the two recombinant classes ( $R_{1}$ and $R_{2}$ ) in a perfectly general way. PI may be defined as the parental class which has the flanking markers which went into the cross with the proximal allele difference. R I may be defined as the recombinant class which combines the proximal flanking marker of the distal allele difference with the distal flanking marker of the proximal allele difference. Thus in the cross $A m^{1}+B \times a+m^{2} b$, the $\mathrm{Pl}$ progeny are $A B$ and the $\mathrm{R} \mathrm{I}$ progeny are $a B$.

\section{MATERIALS AND METHODS}

\section{(a) Organisms}

The majority of the his-I mutants (all those with a number prefixed by the letter $\mathrm{K}$ ) were isolated from Emerson a by filtration enrichment following irradiation of 
TABLE I

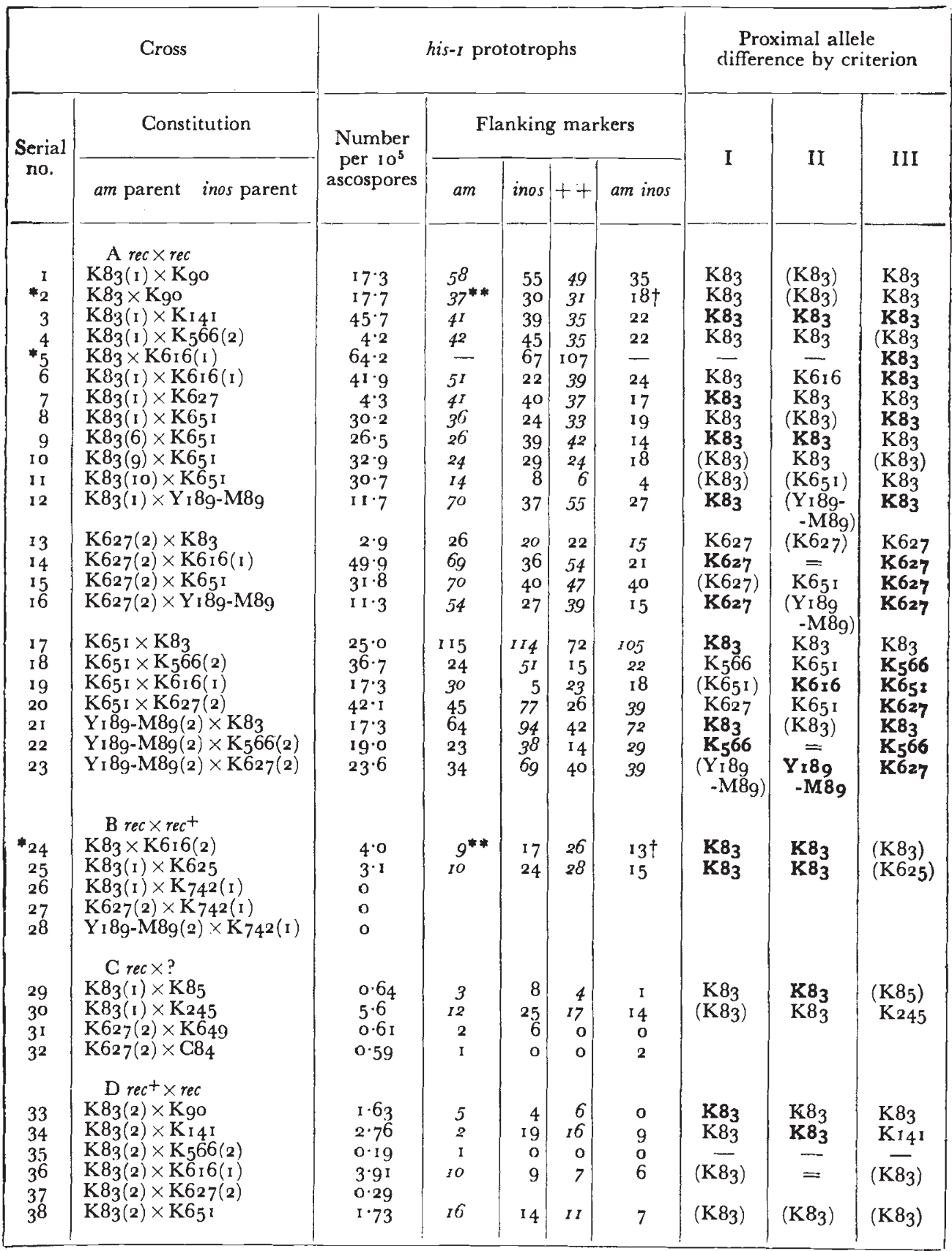

Notes.-(i) All families, except those marked with an asterisk, are of the general form am his- $I^{x} \times$ his-I $^{y}$ inos. (ii) Families $5,46,47,55,56,57,58,62,65$ and 67 are of the general

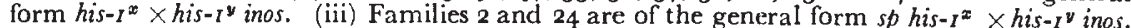
(iv) Where two or more stocks of a given constitution have been used in the crosses, these are distinguished by bracketed numbers placed after the his-I allele number. (v) Constitution of 
TABLE $\mathrm{I}-$ (continued)

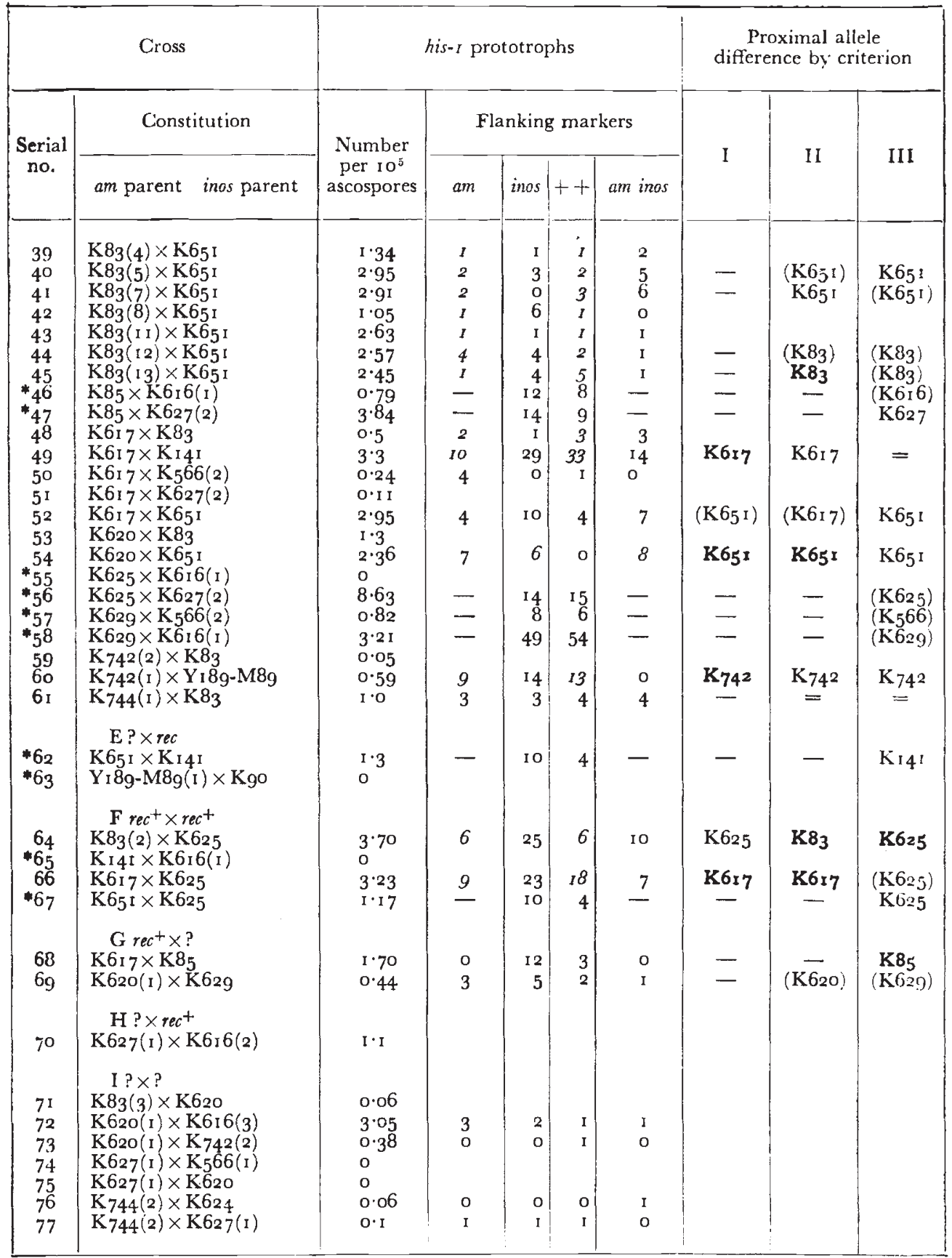

progeny marked ** is $s p$, that of those marked $\dagger$ is sp inos. (vi) Amongst the prototrophs, the $\mathrm{P}_{I}$ and $\mathrm{R}_{\mathrm{I}}$ flanking marker constitutions are identified by the numbers of progenv being in italics. (vii) The levels of significance of identification of the proximal allele difference by criteria I, II and III are indicated by bold face type for P less than 0.05 , while those with $\mathrm{P} .3$ or greater are bracketed. 
conidia with ultra-violet light. These have been listed by Catcheside (1960). Stocks of two other his-I mutants, C8 4 and $\mathrm{Y}_{1} 89-\mathrm{M} 89$, were received from Dr B. Ames and Prof. N. H. Giles, respectively. The amination mutant used was $\mathrm{K}_{\text {I } 34}$, isolated by Catcheside from Emerson a; this is the allele referred to by Dr J. R. S. Fincham as $a m^{6}$. The inositol mutant used was $3740 \mathrm{I}$, originally received from $\operatorname{Dr} \mathrm{G}$. W. Beadle, while spray $\left(\mathrm{B}_{1} 3_{2}\right)$ was donated by $\mathrm{Dr} \mathrm{B}$. R. Smith. The only other stocks which have entered the pedigree are the wild types Emerson $\mathrm{A}$ and Emerson a.

\section{(b) Media}

All stocks were grown and kept on slopes of Vogel's medium (Vogel, I955), suitably supplemented. Crosses were made on slopes of Westergaard's medium (Westergaard and Mitchell, 1947) containing folded strips of filter paper. Tests of cultures for biochemical differences were made on Vogel's medium in which 0.5 per cent. sorbose and 0.1 per cent. sucrose replaced the usual 2 per cent. sucrose; suitable supplements were added.

Appropriate supplements of growth factors were added to the minimal media as follows. For his-I and his-I inos stocks, histidine was added at the rate of 300 $\mathrm{mg} . / \mathrm{l}$. and inositol at $20 \mathrm{mg} . / \mathrm{l}$. For am his-I and am stocks, histidine was used at the rate of $200 \mathrm{mg}$./l. and alanine at $450 \mathrm{mg} . / \mathrm{l}$. Amination mutants will grow on a number of different amino acids and their growth is inhibited by some others (Fincham, 1950). Histidine is inhibitory, so the amount of it was reduced in media for am his- $I$ stocks. However, am mutants can adapt to grow on minimal medium, so glycine at the rate of $1500 \mathrm{mg}$./l. was added to the medium when am was being scored.

\section{(c) Crosses}

Each cross for analysis of prototroph frequency was made in about eight tubes of crossing medium and the ascospores from the different tubes were pooled. Ascospores were collected in sterile water, filtered through butter muslin and, after decantation of excess water, suspended in $0^{\prime} 1$ per cent. agar. The number of spores per ml. of suspension was estimated by counting the number in $0.01 \mathrm{ml}$. samples on slides on which the area covered by the coverslip was divided into 64 squares. The ascospores were screened for prototrophs by a modification of the layer plate technique (Newmeyer, 1954; Murray, 1960). The ascospores in the suspension in 0.1 per cent. agar were activated to germinate by heating at $55^{\circ} \mathrm{C}$. for 60 minutes. Known volumes were then pipetted into $2 \mathrm{ml}$. volumes of 0.5 per cent. agar, kept molten at $45^{\circ} \mathrm{C}$., quickly mixed and evenly spread on plates of Vogel's medium supplemented with inositol and alanine. The plates were incubated at $25^{\circ} \mathrm{C}$. and scanned under a stereomicroscope after 24 hours and 48 hours. Prototrophs were transferred to tubes of Vogel's medium, containing inositol and alanine, and later classified for am and inos. The viability of the ascospores of each cross was determined in samples layer plated on Vogel's medium plus histidine, inositol and alanine.

\section{RESULTS}

When doubly mutant stocks had been prepared, so far as possible in both mating types, crosses of the type am his $-I^{x}+\times+$ his- $I^{y}$ inos, where his- $I^{x}$ and his-I $I^{y}$ designate two his- $I$ alleles, were analysed for their frequency of formation of prototrophs and the prototrophs were then classified for the four combinations of flanking markers, two parental, am + and + inos, and two recombinant, ++ and am inos. The mutant spray was used as proximal marker in two crosses. In a number of crosses there was no proximal marker. The data are summarised in table I, the important parameters in which are (i) the 
frequencies of prototrophs per hundred thousand ascospores analysed in a cross and (ii) the order of the his- $I$ allele differences as deduced from the distribution of the four classes of the flanking marker combinations amongst these prototrophs. These two aspects of the data will be discussed separately.

\section{(a) Prototroph frequencies}

The observed frequencies of prototrophs ranged from o. I (or even zero) to 50 or so per $10^{5}$ ascospores. Of great interest and, initially, a

TABLE 2

Data on prototrophs in crosses of various his-I inos $A$ stocks to two stocks of am $K 8_{3}$ a

\begin{tabular}{|c|c|c|c|c|c|c|c|c|c|c|}
\hline \multirow{3}{*}{$\begin{array}{l}\text { his-1 inos } \mathrm{A} \\
\text { parent }\end{array}$} & \multicolumn{5}{|c|}{$a m \mathrm{~K} 83 \mathrm{a}(1)$} & \multicolumn{5}{|c|}{$a m \mathrm{~K} 83 \mathrm{a}(2)$} \\
\hline & \multirow{2}{*}{$\begin{array}{c}\text { No. per } 10^{5} \\
\text { ascospores }\end{array}$} & \multicolumn{4}{|c|}{ Flanking nuarkers } & \multirow{2}{*}{$\begin{array}{l}\text { No. per } 10^{5} \\
\text { ascospores }\end{array}$} & \multicolumn{4}{|c|}{ Flanking markers } \\
\hline & & $a m$ & inos & ++ & $\begin{array}{l}a m \\
\text { inos }\end{array}$ & & $a m$ & inos & $t+$ & $\begin{array}{l}\text { im } \\
\text { inos. }\end{array}$ \\
\hline $\mathrm{K}_{5} 66$ & 4.2 & 42 & 45 & 35 & 22 & 0.2 & I & o & 0 & o \\
\hline $\mathrm{K} 627(2)$ & 43 & 41 & 40 & 37 & 9 & 0.3 & - & - & - & - \\
\hline Kgo & $17 \cdot 3$ & $5^{8}$ & 55 & 49 & 35 & 1.6 & 5 & 4 & 6 & 0 \\
\hline $\mathrm{K}_{141}$ & 45.7 & 41 & 39 & 87 & $3 \mathrm{I}$ & $2 \cdot 7^{6}$ & 2 & 19 & 16 & 9 \\
\hline K6I6(1) & 41.9 & $5 I$ & 22 & 39 & 24 & $3 \cdot 9$ & 10 & 9 & 7 & 6 \\
\hline K65I & 27.0 & $3^{6}$ & 24 & 33 & 19 & 17 & 16 & 14 & II & 7 \\
\hline K625 & $3 \cdot \mathrm{I}$ & Io & 24 & 28 & 15 & 37 & 6 & 28 & 6 & 10 \\
\hline $\mathrm{K} 83$ & o & & & - & & 0 & - & $\ldots$ & - & - \\
\hline
\end{tabular}

source of embarrassment, werc crosses involving different stocks of the same his-I mutant which gave substantially different frequencies of prototrophs. The analysis had best begin with a pair of stocks of one of these mutants, $\mathrm{K} 83$.

(i) Genetics of prototroph frequency. Two isolates of am $\mathrm{K} 83,{ }^{*}$ both of mating type a, were obtained from a cross of $+\mathrm{K} 83 \mathrm{a} \times a m+\mathrm{A}$. These had the pedigree family numbers 2768 and 2769 and may be designated as $a m \mathrm{~K} 8_{3}(\mathrm{I})$ and $a m \mathrm{~K} 83_{3}(2)$, respectively. Data on the progeny of crosses of both to a series of his-I inos A stocks are gathered together in table 2. It will be noticed in all cases except one, namely the cross with $\mathrm{K} 625$ inos, that am $\mathrm{K} 83$ (I) gives higher prototroph frequencies than does $a m \mathrm{~K} 83(2)$, by a factor of the order of ten. Unfortunately, the cross of am K83(2) with $\mathrm{K} 83$ inos was too infertile to give assurance that the his- $I$ alleles of families 2768 and 2769 were indeed both $\mathrm{K} 83$. However, the data of table 2 would be difficult to explain on the assumption that, say, the his- $I$ allele in family 2769 was not $\mathrm{K} 83$, for in this case the prototroph frequencies of the series of

* In writing the constitutions, only the allele numbers of the his-I mutants will be given . Strictly, the constitution here should be written $a m^{2}$ his-I K83, but since only one mutant allele of $a m$ has been employed, the simpler form of the genotype is unambiguous. 
crosses would not be proportional to those observed in the same series of crosses with family 2768 .

No direct test for allelism was possible since families 2768 and 2769 are of the same mating type. Each was crossed to the wild stock Emerson $\mathrm{A}$ and a number of reisolates of am his- $I$ were obtained. The am his- $I$ reisolates could be of $a$ or $A$ mating type and by this indirect way the his- $I$ alleles in families 2768 and 2769 would have been tested for allelism, had the crosses been sufficiently fertile. Isolates from family 2769 , of a mating type, were also tested by crossing to $\mathrm{K} 65^{\mathrm{I}}$

TABLE 3

his- I prototrophs in crosses of $K 6_{5} I$ inos $A$ with progeny of am $K 8_{3} a(2) \times$ Emerson $A$

\begin{tabular}{|c|c|c|c|c|c|}
\hline \multirow{3}{*}{$\begin{array}{c}a m \mathrm{~K}_{3} \mathrm{a} \\
\text { isolate }\end{array}$} & \multicolumn{5}{|c|}{ his-I prototrophs } \\
\hline & \multirow{2}{*}{$\begin{array}{l}\text { No. per } 10^{5} \\
\text { ascospores }\end{array}$} & \multicolumn{4}{|c|}{ Flanking markers } \\
\hline & & $a m$ & inos & ++ & am inos \\
\hline (4) & $1 \cdot 3$ & I & I & I & 2 \\
\hline (5) & $2 \cdot 95$ & 2 & 3 & 2 & 5 \\
\hline (7) & $2 \cdot 9$ & 2 & 0 & 3 & 6 \\
\hline (8) & $\mathrm{I} \cdot \mathrm{I}$ & I & 6 & I & 0 \\
\hline (II) & $2 \cdot 6$ & I & I & I & I \\
\hline (12) & $2 \cdot 6$ & 4 & 4 & 2 & I \\
\hline (13) & $2 \cdot 5$ & I & 4 & 5 & I \\
\hline (6) & $26 \cdot 5$ & 26 & 39 & $4^{2}$ & 14 \\
\hline (9) & $32 \cdot 9$ & 24 & 29 & 24 & I 8 \\
\hline (10) & 30.7 & I 4 & 8 & 6 & 4 \\
\hline
\end{tabular}

inos. In this way it was found that the progeny of am $\mathrm{K} 83$ a(2) $\times$ Emerson $A$ were showing segregation for frequency of formation of prototrophs (table 3 ), seven giving low values and three high values, the latter being similar to those given by am $\mathrm{K} 8_{3}$ a( $\left.\mathrm{I}\right) \times \mathrm{K} 6_{5} \mathrm{I}$ inos $\mathrm{A}$. It therefore seems clear that a gene difference controlling prototroph frequency is segregating, that presence of the low frequency gene in one parent is sufficient to give low frequencies of prototrophs, while the presence of the high frequency.gene in both parents is necessary to give high frequencies of prototrophs. If the locus concerned is designated recombination, the dominant allele for low frequency may be regarded as the wild type and the recessive allele for high frequency as mutant. Respectively they may be symbolised as $\mathrm{rec}^{+}$and $\mathrm{rec}$.

The rec gene has a large effect upon recombination between his-I alleles. Does it affect recombination between non-alleles or the distribution of flanking markers amongst his- $I$ prototrophs? Does it influence other loci in a similar way?. Work to answer the last question is in progress; it would, no doubt, be surprising if the effects 
of rec were restricted to the his-I locus, but no answer is presently available.

Ascospores from crosses of am $\mathrm{K} 83 \mathrm{rec}$ and am $\mathrm{K} 83 \mathrm{rec}^{+}$to his-I inos rec testers have been analysed for recombination of am and inos (table 4). They were plated out on minimal medium, supplemented

TABLE 4

\begin{tabular}{|c|c|c|c|c|c|c|c|c|}
\hline \multirow{2}{*}{ Parents } & \multicolumn{5}{|c|}{ Progeny } & \multirow{2}{*}{$\begin{array}{l}\text { Recombination } \\
\text { per cent. }\end{array}$} & \multicolumn{2}{|c|}{ Difference } \\
\hline & Total & $a m$ & inos & ++ & àm inos & & $\chi^{2}$ & $\mathrm{P}$ \\
\hline $\begin{array}{l}\text { am } \mathrm{K}_{3} \times \mathrm{K}_{6} 5_{1} \text { inos } \\
\text { (1) } \mathrm{rec} \times \mathrm{rec} \\
\text { (2) } \mathrm{rec}^{+} \times \mathrm{rec}\end{array}$ & $\begin{array}{l}503 \\
631\end{array}$ & $\begin{array}{l}232 \\
295\end{array}$ & $\begin{array}{l}218 \\
276\end{array}$ & $\begin{array}{l}30 \\
23\end{array}$ & $\begin{array}{l}23 \\
37\end{array}$ & $\begin{array}{r}10.54 \\
9.51\end{array}$ & 0.297 & $0.7-0.5$ \\
\hline $\begin{array}{l}a m \mathrm{~K} 8_{3} \times \mathrm{K} 6{ }_{1} 6 \text { inos (1) } \\
\text { (1) } \mathrm{rec} \times \mathrm{rec} \\
\text { (2) } \mathrm{rec}^{+} \times \mathrm{rec}:\end{array}$ & $\begin{array}{l}902 \\
798\end{array}$ & $\begin{array}{l}410 \\
372\end{array}$ & $\begin{array}{l}405 \\
370\end{array}$ & $\begin{array}{l}40 \\
27\end{array}$ & $\begin{array}{l}47 \\
29\end{array}$ & $\begin{array}{l}9.56 \\
7.02\end{array}$ & $\mathrm{I} \cdot 4^{28}$ & $0.3-0.2$ \\
\hline
\end{tabular}

with histidine, alanine and inositol. This gave unselected populations, all members of which carried one of the his- $I$ alleles of the parents. No attempt was made to classify the progeny for the his- $I$ allele, but all were classified for am and inos. The $\mathrm{rec}^{+} \times$rec progenies show slightly smaller frequencies than do the corresponding $r e c \times r e c$ progenies, but the differences are not at all significant.

TABLE 5

Distribution of flanking markers in his- $\mathrm{I}^{+}$progeny of $\mathrm{rec} \times \mathrm{rec}$ and $\mathrm{rec}^{+} \times \mathrm{rec}$ crosses of $K_{3}{ }_{3}$ and $K 6_{5} I$.

\begin{tabular}{|c|c|c|c|c|c|}
\hline Cross & am & inos & ++ & am inos & Total \\
\hline$a m \mathrm{~K}_{3} \mathrm{rec}^{+} \times \mathrm{K} 651$ inos rec & 28 & 33 & 26 & 23 & $1 \mathrm{ro}$ \\
am K83 rec $\times \mathrm{K} 65 \mathrm{I}$ inos rec & 100 & 100 & 105 & 55 & 360 \\
am K651 rec $\times \mathrm{K} 83$ inos rec & 115 & 114 & 72 & 105 & 406 \\
\hline
\end{tabular}

Data on the distribution of flanking markers amongst selected his-I prototrophs are contained in tables $\mathrm{I}, 2$ and 3 . In general the data for the $\mathrm{rec}^{+} \times \mathrm{rec}$ crosses are too meagre to permit reliable comparison. Such differences as are shown in table 2 are inconsistent from one pair of crosses to another and similar differences are shown (table 3) in the series of am $\mathrm{K} 83 \mathrm{rec}^{+}$crosses to $\mathrm{K} 6_{5} \mathrm{I}$ inos rec. The data of the $\mathrm{K}_{3} \times \mathrm{K}_{5}$ I crosses are summed in table 5. The rec $\times$ rec crosses are seen to show significant asymmetries, while the $\mathrm{rec}^{+} \times \mathrm{rec}$ crosses do not. However, more data are needed before this can be accepted as a reasonable generalisation. 
(ii) Classification of stocks for rec. It has been shown that the stocks so far considered may be classified for rec. Using them, attempts may be made to identify the constitutions of further stocks, with a view to grouping the prototroph data into high and low frequency sets. Besides am $\mathrm{K} 83(2)$, which is $\mathrm{rec}^{+}, \mathrm{K} 625$ inos is the only other $\mathrm{rec}^{+}$ stock amongst those listed in table 2, all the rest being rec. These, then, provide a set of testers of known constitution with respect to recombination.

Strictly, to conclude whether a given stock of a his-I mutant is $\mathrm{rec}^{\div}$or rec requires measurement of the prototroph frequencies in crosses of it to known $\mathrm{rec}^{+}$and rec stocks, carrying another suitable his-I allele, used as testers. A rec ${ }^{+}$stock of his- ${ }^{x}$ would give equal, low values of prototroph frequency with his- $I^{y} \mathrm{rec}^{+}$and his- ${ }^{y} \mathrm{rec}$ testers. A rec stock of his- $I^{x}$ would give different values with the testers; the value would be relatively low with his- ${ }^{y} \mathrm{rec}^{+}$and relatively high with his- $I^{y}$ rec. In general, such data are not available, because a sufficient range of pairs of tester stocks has not so far been included in the crosses. However, it is worth while to attempt to deduce the constitutions of the stocks, with the help of collateral evidence, so far as is possible.

Of course, Emerson A is rec, since segregation is shown in its cross with the low frequency stock am K8 $3 \mathrm{a}(2)$. The two stocks am $\mathrm{K} 627(2)$ and $\mathrm{K} 83$ inos are both $r e c$ because their cross gives a frequency of prototrophs comparable to that of am $\mathrm{K} 83(\mathrm{I}) \times \mathrm{K} 627$ inos (2). Likewise, $\mathrm{K} 83$ is rec, because its cross gives a high frequency with K6I6 inos (I). Indeed the frequency is higher than in the cross am $\mathrm{K} 83$ ( $\mathrm{I}) \times \mathrm{K} 6 \mathrm{r} 6$ inos (I), namely $64 \cdot 2 \pm 4 \cdot 74$ per I $^{5}$ compared with $41 \cdot 9 \pm 3 \cdot 23$ per ${ }^{5}{ }^{5}$. The difference, $22 \cdot 3 \pm 5 \cdot 74$, is highly significant $(\mathrm{P}<\mathrm{O} .00 \mathrm{I})$, indicating that other factors, perhaps of genetic origin, influence prototroph frequencies, though in a less spectacular way than does rec.

The stocks am Yi89-M89(2) and Yi89-M89 inos must be rec. Since the cross am Yi89-M89(2) $\times \mathrm{K}_{90}$ inos gave a zero frequency of prototrophs, the sites of $\mathrm{Y}_{1} 89-\mathrm{M} 89$ and K9o must be very close together. However, the prototroph frequencies in the crosses am K83 (I) XY ${ }_{1} 89-\mathrm{M} 89$ inos and am $\mathrm{Y}_{1} 89-\mathrm{M} 89(2) \times \mathrm{K} 83$ inos are both similar to the frequency in am $\mathrm{K} 8_{3}(\mathrm{I}) \times \mathrm{K}_{90}$ inos. By a comparable argument am $\mathrm{K} 65 \mathrm{I}$ also appears to be rec. In particular, it gives similar prototroph frequencies in crosses to $\mathrm{K} 83$ inos and $\mathrm{K} 627$ inos (2), respectively, as does the known rec stock $\mathrm{K} 65 \mathrm{I}$ inos with known rec stocks of $\mathrm{K} 83$ and $\mathrm{K} 627$. The stock $s p \mathrm{~K} 83$ is also rec.

The above identifications probably exhaust all of the rec stocks in the data of table I. Of course, reliance cannot be placed on the actual frequency of prototrophs in an isolated cross, since even two rec parents may have sites of difference so close together that recombination is rare. The observed range of prototroph frequencies in $\mathrm{rec} \times \mathrm{rec}$ crosses extends down to about 3 per $\mathrm{IO}^{5}$, while in $\mathrm{rec}^{+} \times$rec crosses it 
extends up to about 4 per $10^{5}$ according to the data in table 2. The lower values in $r e c \times r e c$ crosses overlap with the higher values in $\mathrm{rec}^{*}$ $\times$ rec and $\mathrm{rec}^{+} \times \mathrm{rec}^{+}$crosses. Theoretically, the whole range to be found in $\mathrm{rec}^{+}$crosses should be included in the lower tenth or so of the whole range to be found in rec $\times$ rec crosses.

Therefore, one indirect, though not perfectly conclusive, method of recognising a $\mathrm{rec}^{+}$stock would be by the apparent compression of the range shown in crosses of it to rec stocks known to have his-I mulants well dispersed in the range. By this means, one may conclude that the following stocks are $\mathrm{rec}^{+}$: am K6r7, am K620(2), K620 inos, am $\mathrm{K}_{742}$ ( $\mathrm{I}$ and 2), $\mathrm{K}_{742}$ inos (1), K629, K85, K625 and $\mathrm{K}_{14}$ I. There is much less confidence in the suggestion that $\mathrm{K} 85$ inos, $\mathrm{K} 629$ inos, $\mathrm{K} 65 \mathrm{I}$ and $\mathrm{am} \mathrm{K}_{744}$ (I) are also $\mathrm{rec}^{+}$. Nothing very definite can be said about the few remaining stocks used in a few crosses. In fact, only three of the his-I mutants were definitely used in both rec and $\mathrm{rec}^{+}$stocks. These are $\mathrm{K}_{3}, \mathrm{~K}_{1} 4 \mathrm{I}$ and $\mathrm{K} 6{ }_{1} 6$.

Twenty-four combinations of amination with eight different his-I mutants have been made. Omitting the ten stocks of am K83 isolated from am $\mathrm{K}_{3}(2) \times$ Emerson $\mathrm{A}$, there are $4 \mathrm{rec}, 6 \mathrm{rec}^{+}$and 4 uncertain; including the ten am $\mathrm{K} 83$ isolates there are $7 \mathrm{rec}, \mathrm{I}_{3} \mathrm{rec}^{+}$and 4 uncertain. Similarly, there are twenty-two combinations of inos with seventeen his-I mutants; 8 are rec. 4 are $\mathrm{rec}^{+}, 2$ more are probably $\mathrm{rec}^{+}$and 8 cannot be determined. Of the stocks of his-I mutants used unaccompanied by am or inos, 4 are definitely $\mathrm{rec}^{+}$, one is probably $\mathrm{rec}^{+}$and one is rec. The last of these, $\mathrm{K} 83$, was not the original mutant isolated from Emerson a, but a reisolate from the original stock crossed to Emerson A. It, therefore, appears highly probable that Emerson a carries the gene $\mathrm{rec}^{+}$, that all the mutants originally carried $\mathrm{rec}^{+}$and that the introduction of rec has occurred by outcrossing.

Having identified the constitutions of the parental stocks with respect to $\mathrm{rec}^{+}$and $\mathrm{rec}$ the set of 77 crosses available may be arranged in groups of high and low frequency crosses, as has in fact been done in table I. Group A includes all of the crosses giving high frequencies of prototrophs. Groups B, D and F include the crosses giving low frequencies. Groups C, E, G, H and I include all the crosses in which one or both of the parents is of unknown constitution with respect to rec; these cannot be used in mapping. In general, within the high and low groups, respectively, the prototroph frequencies shown by crosses between different stocks of the same pair of his- $I$ alleles are similar and only sometimes significantly different. Thus, crosses 8, 9, Io and I i involving $\mathrm{K} 83 \times \mathrm{K} 6_{5}$ I are consistent, not even the difference of $6.5 \pm 3.95$ between the least and greatest (crosses 9 and 10 ) being significant. Neither is the difference of $7 \cdot 9 \pm 3.88$ between families Io and 18 significant. Apart from the case of crosses 5 and 6 previously noted (p. 246), the only significant differences involve crosses I 6 and 21 (difference $10.3 \pm 3 \cdot 78, \mathrm{P}<\mathrm{O} \cdot \mathrm{OI}$ ) and crosses $\mathrm{I} 2$ and 22 (difference $5 \cdot 6 \pm \mathrm{I} \cdot 33, \mathrm{P}<\mathrm{O} \cdot 000 \mathrm{I})$. 
(iii) Mapping by prototroph frequencies. All the data for each pair of his-I alleles may be combined, separately for the high and low frequency crosses, but omitting those crosses involving stocks whose rec constitution is uncertain or unknown. The estimates of prototroph

TABLE 6

Estimates of prototroph frequencies, per $10^{5}$ ascospores, in his-1 crosses

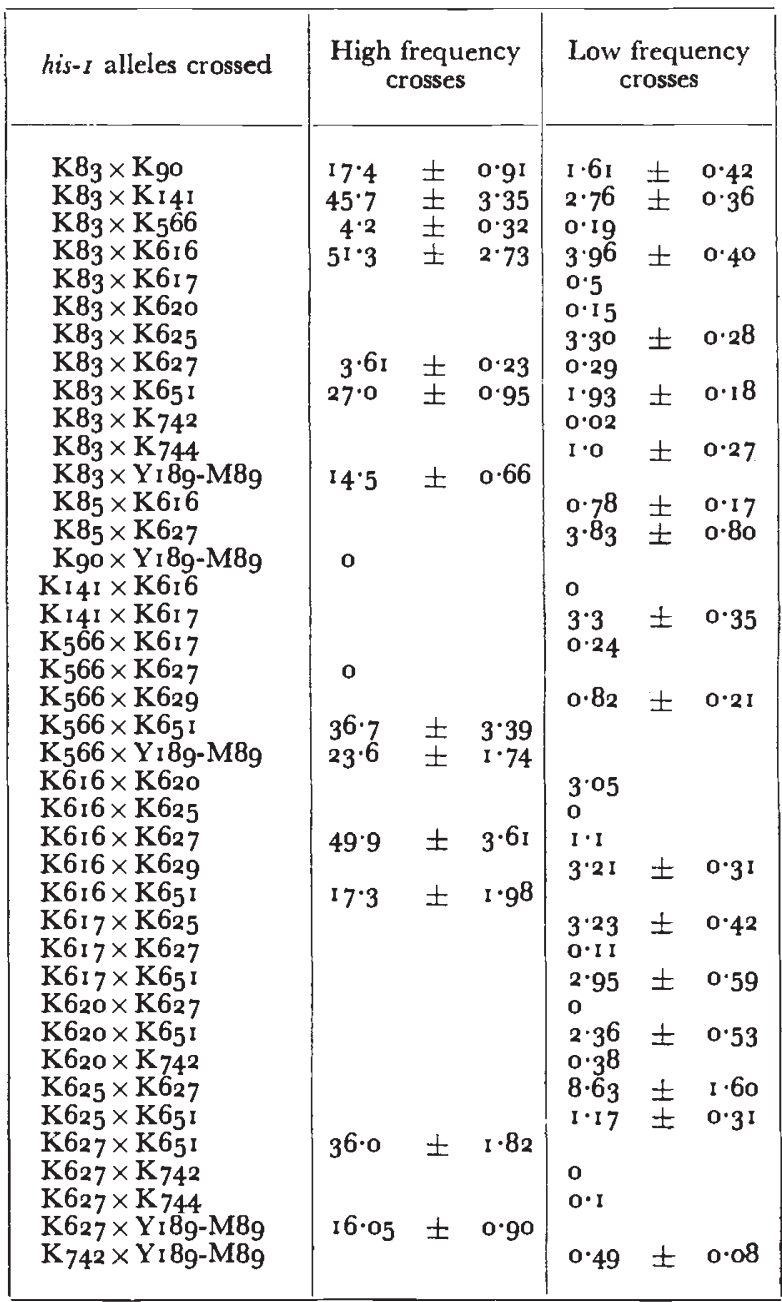

frequencies, which may be used for the construction of a fine structure map, are summarised in table 6. Maps based on the high and low frequency data are shown in fig. 2. It will be seen that the two maps agree reasonably well, in respect of the order of the allelic differences and their relative spacing. However, the scales of the maps are greatly different, by a factor of about 14.5 , rather than the tenfold difference as it has been described roughly in earlier paragraphs. 
(b) Distribution of flanking markers

The data upon the distribution of the flanking markers, generally $a m$ and inos, are listed in table $\mathrm{I}$, which also contains the order of the his- $I$ allele differences as inferred according to the three criteria explained in the introduction (p. 4). The orders are expressed in terms of the proximal allele difference, for every case where at least

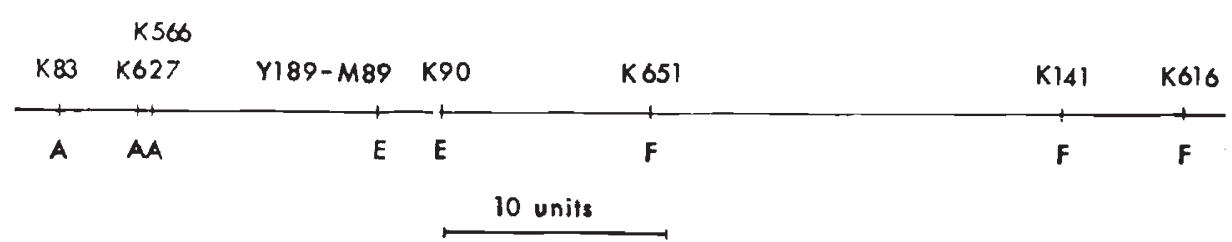

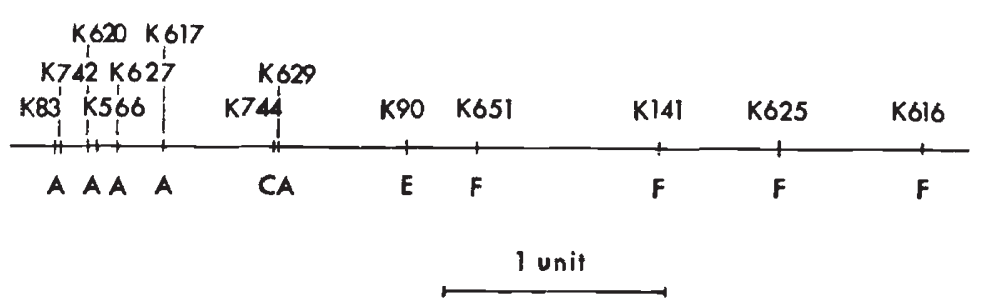

FIG. 2.-Genetic maps of the his- $I$ gene based on prototroph frequencies in progeny of crosses; (a) high frequency crosses, (b) low frequency crosses. The letters below the maps designate the complementation group to which the individual alleles belong.

ten prototrophs are concerned in the comparison made. For each entry, some indication is given of the statistical reliability of the orders. Those significant at the 5 per cent. level ( $P=0.05$ or less) are shown in bold type, while those with a $\mathbf{P}$ value of 0.3 or greater are enclosed in brackets. The PI and RI classes are identified in columns 6-9 of table I by italics; $P_{1}$ occurs in either the $a m$ or inos columns and $\mathrm{R} \mathrm{r}$ in either the ++ or am inos columns.

Taken as a whole, these data indicate an order of the allele differences (fig. 3) which is consistent with the order derived from comparison of prototroph frequencies (fig. 2). The consistency of the order derived from consideration of the distribution of the flanking markers is most readily grasped by plotting the data by means of lines joining the positions of the allele differences, the proximal position being marked by an arrow. In fig. 3, only the orders significant at the 5 per cent. level are used and the order used is that which gives the least number of discrepancies. Criterion I gives a high degree of consistency and this is maintained even if the orders based on less significant data are also included. The other two criteria are less consistent, criterion III giving better agreement than does criterion II.

\section{(c) Comparison of genetic and complementation maps}

Reference has already been made to the supposition that the complementation map of his-I may be two-dimensional (Catcheside, 
I 96o). However, it was a matter of some doubt, mainly because certain combinations of mutants, namely $\mathrm{K} 626+\mathrm{K}_{745}$ and $\mathrm{K} 626$ $+\mathrm{K} 85$, formed heterocaryons erratically. It was noted that when conidia of these mutants were mixed, growth could either occur quickly or after a long lapse of time (up to IO-I 2 days) or not at all. When growth was established, these heterocaryons continued to grow
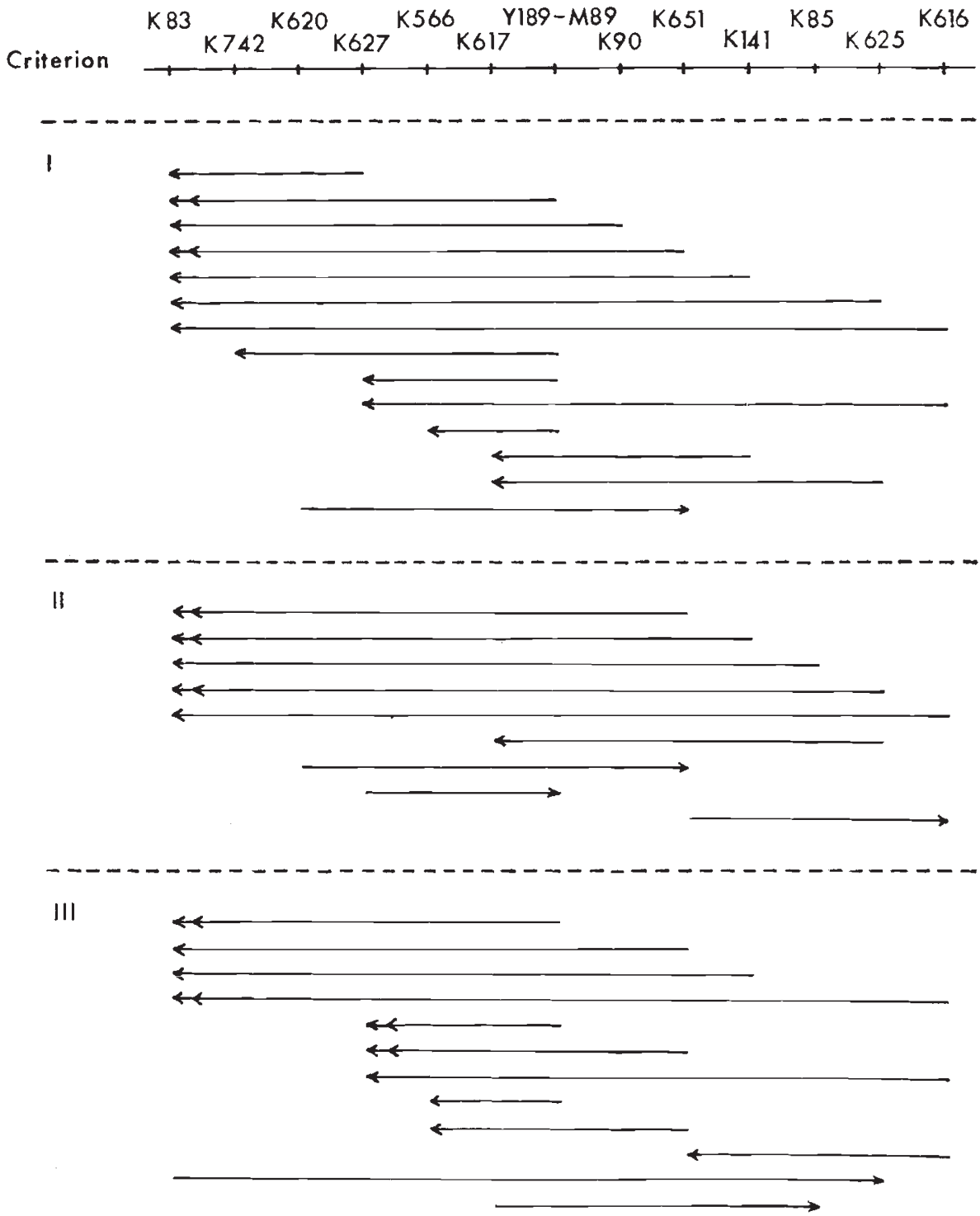

Fig. 3.-Diagrams to show the orders of his- 1 allele differences, as inferred from distribution of flanking genetic markers amongst prototrophs, superimposed on the map order deduced from prototroph frequencies. Each arrow narks the proximal one of the pair of alleles joined by a given line. I, II and III are the three criteria used (see pp. 238・9). Each arrow point represents one cross. 
vigorously. Their heterocaryotic conidia germinated without delay and, in their turn, formed cultures which grew vigorously on minimal medium. Both of the original components could be recovered from the heterocaryons.

These combinations have been further studied by introducing additional nutritional mutants* into $\mathrm{K} 626, \mathrm{~K}_{745}$ and $\mathrm{K} 85$ so that forced heterocaryons, cultured on minimal medium supplemented with histidine, could be prepared. In turn, these were tested for their ability to grow on minimal medium, with the finding that generally all such heterocaryons involving $\mathrm{K} 626+\mathrm{K} 85$ or $\mathrm{K} 626+\mathrm{K} 745$ were unable to grow on minimal medium. Hence, it must be concluded that these combinations do not complement.

It was found that the forced heterocaryons sometimes began to grow, much as did some of the mixed inoculations in the original experiments. Some of these cultures were analysed by plating samples of their conidia on minimal medium supplemented with histidine and the other compounds needed by the original constituents of the heterocaryon. The resulting colonies were analysed for their nutritional requirements. In this way it was found that the growth of the heterocaryons in the absence of histidine was due to reversion of $\mathrm{K} 626$ to prototrophy. Thus, conidia from a heterocaryon formed between K626, 36ro4 and $\mathrm{K}_{745}, \mathrm{Y}_{2198}$, 5531, found capable of growth on minimal medium, gave colonies of four kinds: (i) requires histidine +methionine, (ii) requires histidine + tryptophan + pantothenic acid, (iii) grows in minimal medium, (iv) requires methionine. The first two are the parental constituents, the third could be their heterocaryon but it is not, while the fourth is a parental constituent in which K626 has reverted. The heterocaryotic colonies which can grow on minimal medium always contain the $\mathrm{K} 6{ }_{2} 6$ reversion as one component.

However, the performances of forced heterocaryons, as well as further repeated experiments, confirm the original finding that the combination of $\mathrm{K} 624+\mathrm{K} 626$ does complement slightly. The heterocaryon attains, on minimal medium, about 25 per cent. of the normal amount of growth shown on supplemented medium or by the wild type on minimal medium. Hence the his-I mutants fall into six groups rather than the eight groups previously reported. The members of one of these groups, comprising nearly all of the his-I mutants, are not complementary with any his-I mutants. The complementation map is shown in fig. 4. The relation between the complementation groups now recognised and those listed in Catcheside ( 1960$)$ is set out in table 7 .

We are now in a position to compare the genetic and complementation maps. In fig. 2, it will be seen that all mutants belonging to any one complementation group are in an individual region of the genetic map, unmixed with mutants belonging to any other complementation

* The nutritional markers used in these experiments were $m e-3(36104), t r y-3(\operatorname{td} \mathbf{r})$ and $\operatorname{try}-\frac{1}{4}(Y 2198)$ accompanied by pan-I (5531). 
group. The A group, of non-complementing mutants, is in a proximal section of the genetic map, amounting to about a fifth of the known length of the map. These are followed by a section, about a fifth of the map, containing the $\mathrm{E}$ mutants and finally by the $\mathrm{F}$ mutants in the distal half to three-fifths of the map. Only the $\mathrm{C}$ mutant, $\mathrm{K}_{744}$, may be amongst the more distal of the A mutants. However, its actual position cannot be determined with any certainty on the available evidence.

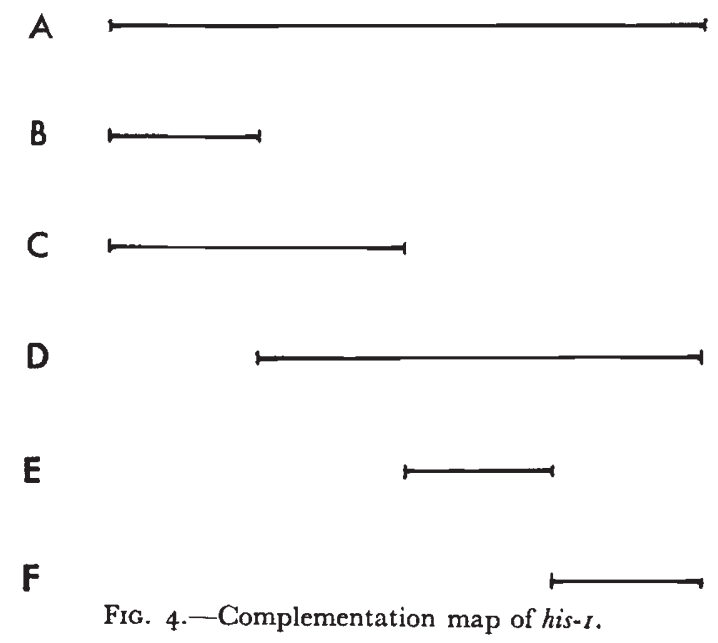

TABLE 7

Synonymy of complementation groups of his-1 mutants mapped genetically

\begin{tabular}{|c|c|c|}
\hline Present group & $\begin{array}{l}\text { Previous groups } \\
\text { (Catcheside, 196o) }\end{array}$ & Mutants mapped genetically \\
\hline A & A & \multirow{4}{*}{$\begin{array}{l}\mathrm{K}_{3}, \mathrm{~K}_{245}, \mathrm{~K}_{566}, \mathrm{~K}_{11}, \mathrm{~K} 620 \\
\mathrm{~K}_{27}, \mathrm{~K} 649, \mathrm{C} 84 \\
\mathrm{~K}_{744} \\
\mathrm{~K}_{90}, \mathrm{~K}_{742}, \mathrm{Y}_{1} 89-\mathrm{M} 89 \\
\mathrm{~K}_{5}, \mathrm{~K}_{14}, \mathrm{~K}_{1} 6, \mathrm{~K}_{25}, \mathrm{~K}_{51}\end{array}$} \\
\hline B & B & \\
\hline C & C & \\
\hline E & $\begin{array}{l}\text { E, G } \\
\text { F, H }\end{array}$ & \\
\hline
\end{tabular}

\section{DISCUSSION}

The primary purpose of the study of recombination between his- I mutants was to construct a genetic map to show the relative positions of the allelic differences, with the intention of making a comparison with the complementation map. It turns out that the relationship, so far as it is established, is a very simple one with mutants belonging to the same complementation group being grouped together in a distinct segment of the genetic map. Beyond the demonstration of a very good correlation of the two maps, there is only one special property. The notable feature, compared with other loci, is that the 
non-complementing mutants appear to be concentrated at one end, the proximal end, of the map. The more usual situation, encountered in pan-2 (Case and Giles, 1958), me-2 (Murray, 196o, I963) and his-5 (Smith, 1965), is for the non-complementing mutants to be spread all along the genetic map, mingled with the sites of difference of the complementing mutants. His- $I$ may not be unique in its proximal localisation of the sites of non-complementing mutants, for his-3 shows a similar distribution (Catcheside, 1964), though with some of the kinds of complementing mutant apparently mingling with the non-complementing ones.

The gene which affects the frequency of recombination between alleles is of unusual interest. Although the mechanism of recombination at the molecular level is a matter of fundamental importance, theories which take account of the molecular architecture of deoxyribonucleic acid and of the time of its replication with respect to the time of genetic recombination at meiosis are only of very recent proposal (Holliday, 1962, 1964; Whitehouse, 1963; Hastings and Whitehouse, I964; Whitehouse and Hastings, I 964). There is no need at this juncture to explain the various theories beyond saying that they include, variously, separation over short distances of the two chains of a DNA molecule, the breakage of one or both of these chains so separated, the formation of segments of hybrid DNA, the excission of mispaired bases or of segments of a DNA chain, and the reconstruction of segments of the DNA chain using information in the other chain. Many of these events involve chemical reactions, either the dissolution of bonds in breakage and excission or the formation of bonds in processes of reconstruction. It is characteristic of organisms that chemical processes of these kinds are catalysed by enzymes and that each kind of enzyme is structurally specified by a gene and is subject to control, directly or indirectly, by other gene systems. It is in these terms that some attempt must be made to understand the possible ways in which the gene rec acts.

In the first place, it appears that rec is fully recessive to $\mathrm{rec}^{+}$, one dose of $\mathrm{rec}^{+}$being as effective as are two doses. It is an axiom that dominance implies that the gene product is metabolically active. Hence, there are two general modes of action of $\mathrm{rec}^{+}$, namely either it determines the structure of an enzyme which catalyses one of the many different events which make up genetic recombination or else it determines the structure of a regulatory substance which either activates or represses one of these enzymes. In practice, it would probably be difficult to distinguish between production of an enzyme and activation of an enzyme. Hence, consideration may be restricted to comparison of $\mathrm{rec}^{+}$having a metabolic activity as compared with it repressing a metabolic activity. Furthermore, discussion may assume that the effects of the gene are general, rather than restricted in action to alleles of his-I; this assumption is being subjected to test.

The effects of substitution of rec for $\mathrm{rec}^{+}$appear to be primarily a 
more than ten-fold increase in the formation of prototrophs by crosses between allelic genes. This is not accompanied by any corresponding increase in recombination between neighbouring non-allelic genes. It does appear to be accompanied by some increase in the degree of asymmetry in the distribution of flanking markers amongst the prototrophs which are selected, but this effect is by no means firmly established. The most likely activity controlled by $\mathrm{rec}^{+}$may well be some kind of monitoring enzyme which corrects faults which appear in the DNA. If it were an enzyme postulated to induce breaks in the nucleotide strands to allow hybrid DNA to form, one would expect the high frequency to be dominant to low frequency. If it were an enzyme which excised a particular kind of mispaired base one would again expect high frequency to be dominant to low frequency. Moreover, there should be other associated effects, consequent upon the specificity of the enzyme for a particular aspect of a particular defect. The direction of the correction may in the normal be expected to go either way, but in the mutant there would be a strong bias in one of these ways, leading most likely to a change in the distribution of flanking markers amongst the prototrophs. Further, the effect should be limited to those alleles, or pairs of alleles, capable of generating the particular defect in hybrid DNA.

The most plausible suggestion that can be made is that the effect is on the breakage process. For hybrid DNA to be formed, a break must exist for at least a short period of time. If there were a variation in the efficiency of a repair process, particularly one repairing breakages, the less efficient process would permit the more frequent formation of hybrid DNA and so the possibility of occurrence of the other events which lead to allelic recombination. This might be a means whereby the frequency of allelic recombination could change quite markedly without the distribution of flanking markers amongst the prototrophs being necessarily altered relatively. However, it is difficult to see why there should, at the same time, be no strong effect on the recombination of non-allelic genes, unless allelic and non-allelic recombination occur by effectively distinct mechanisms.

A remarkable feature of the recombination data collected from various fungi is the great variability which exists not only between species, but also between genes in the same species and between the same pairs of alleles. In the main, interest has been concentrated on fine structure mapping and on the mechanism of "conversion". In these regards, the variability observed has seemed more of a nuisance than a possible source of information about mechanisms (Catcheside, Jessop and Smith, 1964). The variability appears to concern all aspects of the recombination. Referring only to the more striking data in the literature, variation is seen to be manifested in the following ways: (i) prototroph frequency, e.g. for the pan-2 alleles 5 and 3 (Case and Giles, 1958), (ii) the proportion of prototrophs showing recombination of the flanking markers, i.e. variation in the fraction $\mathrm{R}_{\mathrm{I}}+\mathrm{R}_{2} / \mathrm{T}$, 
e.g. the pan-2 alleles 5 and 3 and the inos alleles $3740 \mathrm{I}$ and $6400 \mathrm{I}$ (Giles, I956), (iii) the ratio of $\mathrm{R}_{\mathrm{I}}$ and $\mathrm{R}_{2}$ types of prototrophs, e.g. the cys alleles 80702 and 4840 I (Stadler, I959), the am alleles 2 and 3 (Pateman, I960) and the inos alleles $3740 \mathrm{I}$ and $6400 \mathrm{I}$, (iv) the ratio of $\mathrm{P}_{1}$ and $\mathrm{P}_{2}$ types of prototrophs, e.g. the inos alleles previously mentioned. Indeed, in the case of the inos alleles $3740 \mathrm{o}$ and $6400 \mathrm{I}$, the variations shown by the distribution of the flanking marker combinations are correlated with the variation in prototroph frequency. Some of the variations in the statistical characteristics of prototrophs are accompanied by differences in frequencies of recombination between non-allelic genes, but not apparently in any definitely systematic fashion. Nothing is known of the genetics of the variations in recombination frequency shown by non-allelic genes, e.g. the second division segregation frequencies shown by lys-5 (= asco) (Stadler, I956).

In addition to the variations mentioned, which are detectable amongst the prototrophs screened from samples of individual ascospores, other sorts of variation are detectable in asci examined whole. Some alleles, it seems, show appreciable frequencies of post-meiotic segregation, while others do not. It has not so far been shown whether this is exclusively a property of the particular allele itself or whether there may also be genetic differences of more general effect.

It is clear that there is an ample field for the exploration of genetic and other variations which affect recombination. A full understanding of these appears to be essential to any complete description of the molecular processes which give rise to genetic recombination.

\section{SUMMARY}

A fine structure map of some of the allelic differences found in mutations of the his-I gene has been constructed. The data used comprise the frequencies of prototrophs produced by allelic crosses and the distribution of flanking markers amongst these prototrophs. The genetic map and the complementation map of his- $I$ correlate well as regards the complementing mutants. A notable feature, compared with other loci, is that the non-complementing mutants appear to be concentrated at the proximal end of the genetic map. They are not mingled with the majority of the complementing mutants.

A gene, recombination, which affects the frequency of recombination between alleles, has been found. The frequencies of prototrophs formed in allelic crosses are caused by it to alter by a factor greater than ten. The low frequency allele, $\mathrm{rec}^{+}$, is dominant to the high frequency allele, rec. The gene affects non-allelic recombination to a very small extent if at all. The significance of this gene for an understanding of genetic recombination at the molecular level is discussed.

Acknowledgment.-The work was supported by a research grant awarded by the Department of Industrial and Scientific Research. 


\section{REFERENCES}

AMES, B. N. 1957. The biosynthesis of histidine: D erythro-imidazole glycerol phosphate dehydrase. 7. biol. Chem., 228, 131-1 43.

CASE, MARY E., AND GILES, N. H. 1958. Recombination mechanisms at the pan-2 locus in Neurospora crassa. Cold Spring Harb. Symp. Quant. Biol., 23, I $19+135$.

CATCHeSIDE, D. G. 196o. Complementation among histidine mutants of Neurospora crassa. Proc. Roy. Soc. Lond., B 533 , I 79-194.

CATCHESIDE, D. G. 1964. Allelic complementation. In "Subunit Structure of Proteins ". Brookhaven Symp., I7, I-14.

CATCHESIDE, D. G., JESSOP, ADRIENNE, P., AND SMITH, B. R. 1964. Genetic controls of allelic recombination in Neurospora. Nature, 202, 1242-1 243.

FINCHAM, J. R. S. I950. Mutant strains of Neurospora deficient in aminating ability. 7. biol. Chem., $183,6 \mathrm{I}-73$.

GILES, N. H. I956. Forward and back mutation at specific loci in Neurospora. Brookhaven Symp. Biol., 8, 103-125.

hASTINGS, P. J., AND WHITEHOUSE, H. L. K. 1964 . A polaron model of genetic recombination by the formation of hybrid DNA. Nature, 201, 1052-1054.

HOLLIDAY, R. 1962. Mutation and replication in Ustilago maydis. Genet. Res.. 3, $472-486$.

Holliday, R. 1964. A mechanism for gene conversion in fungi. Genet. Res.. 5, 282-304.

MURRAY, NOREEN E. I96o. Complementation and recombination between methionine-2 alleles in Neurospora crassa. Heredity, 15, 207-2 I 7 .

MURRAY, NOREEN E. 1963. Polarized recombination and fine structure within the me-2 gene of Neurospora crassa. Genetics, 48, I I63-1 183 .

NEWMEYER, DOROTHY. 1954. A plating method for genetic analysis in Neurospora. Genetics, 39, 604-6 18.

PAtEman, J. A. I960. High negative interference at the am locus in Neurospora crassa. Genetics, 45, 839-846.

sмitн, в. R. 1965. Interallelic recombination at the his-5 locus in Neurospora crassa. Heredity, 20, 257-276.

StADleR, D. R. 1956. A map of linkage group VI of Neurospora crassa. Genetics, $4 x$, 528-543.

STADleR, D. R. 1959. Gene conversion of cysteine mutants in Neurospora. Genetics, $44,647-655$.

STRICKLAND, W. N., PERKINS, D. D., AND VEATCH, C. C. 1959. Linkage data for group V markers in Neurospora. Genetics, 44, 1221-1226.

Vogel, H. J. 1955. A convenient growth medium for Neurospora. Microbial Genet. Bull., I3, 42.

WestergaARD, M. AND mitchell, H. K. 1947. Neurospora V. A synthetic medium favouring sexual reproduction. Amer. F. Bot., 34, 573-577.

Whitehouse, H. L. K. I963. A theory of crossing-over by means of hybrid deoxyribonucleic acid. Nature, 199, I 034 -1 040.

WHITEHOUSE, H. L. K., AND haSTINGS, P. J. 1965. The analysis of genetic recombination on the polaron hybrid DNA model. Genet. Res. (in press). 